\title{
Cognitive impairment in multiple sclerosis: diagnosis and monitoring
}

\author{
Virginia Meca-Lallana ${ }^{1}$ (1) • Francisco Gascón-Giménez ${ }^{2} \cdot$ Ricardo C. Ginestal-López $^{3} \cdot$ Yolanda Higueras $^{4}$. \\ Nieves Téllez-Lara ${ }^{5}$ • Joan Carreres-Polo ${ }^{6}$ - Sara Eichau-Madueño ${ }^{7}$ Jesús Romero-Imbroda ${ }^{8}$ - Ángela Vidal-Jordana ${ }^{9}$. \\ Francisco Pérez-Miralles ${ }^{10}$
}

Received: 31 May 2020 / Accepted: 4 March 2021 / Published online: 1 April 2021

(C) The Author(s) 2021

\begin{abstract}
Introduction Cognitive impairment (CI) has a prevalence of $45-70 \%$ in people with multiple sclerosis (MS), producing a negative impact on their quality of life, personal life, and work. Early detection of CI has become an important aspect to be considered for an adequate follow-up, to optimize social adaptation and to implement specific cognitive rehabilitation strategies. The aim of this work is to propose a suitable cognitive evaluation of patients with MS based on available and efficient tools for diagnosis and monitoring purposes well supported by literature review and clinical experience.

Methods A multidisciplinary panel of professionals from the field of neurology, neuropsychology, and neuroimaging performed a literature review of the topic of cognitive impairment assessment. This was combined and completed with their clinical experience to produce a set of recommendations.

Results Some limitations to cognitive evaluation are described: shortage of time and resources during the neurology consultation, scarceness or absence of specialized professionals' availability, importance of tests adaptation, and doubts about its use to define therapeutic efficiency. We recommend a baseline and annual screening evaluation, and we suggest a baseline and periodic neuropsychological assessment. The latter ought to change to a recommendation with the presence of either positive screening test, or subjective to cognitive complaints, screening-test results and patient or family report mismatch, or in specific social/work situations.

Conclusions Cognitive evaluation should be performed on all patients diagnosed with MS and throughout follow-up. It is necessary to support the creation of multidisciplinary MS teams to optimize the evaluation and follow-up of MS patients.
\end{abstract}

Keywords Multiple sclerosis · Cognitive dysfunction $\cdot$ Neuropsychological tests $\cdot$ Neurophysiological monitoring

\section{Introduction}

Cognitive psychology defines several cognitive processes as the focus of neuropsychological evaluation, such as language,

Virginia Meca-Lallana

virmeca@hotmail.com

1 Unidad de Enfermedades Desmielinizantes, Servicio de Neurología, Hospital Universitario de La Princesa, Madrid, Spain

2 Unidad de Esclerosis Múltiple, Servicio de Neurología, Hospital Clínico Universitario, Valencia, Spain

3 Servicio de Neurología, Hospital Clínico San Carlos, Madrid, Spain

4 Instituto de Investigación Sanitaria del Gregorio Marañón, Hospital Gregorio Marañón, Madrid, Spain

5 Servicio de Neurología, Hospital Clínico Universitario, Valladolid, Spain thinking, attention, memory, and emotions. Cognitive impairment (CI) is a common symptom in multiple sclerosis (MS) with a prevalence of 50-60\%. It influences the patients' quality of life, work, and social functioning [1-3]. The most

6 Servicio de Radiología, Hospital Universitari i Politècnic La Fe de Valencia, Valencia, Spain

7 Servicio de Neurología, Hospital Universitario Virgen Macarena, Sevilla, Spain

8 Servicio de Neurología, Hospital Regional Universitario de Málaga, Málaga, Spain

9 Servicio de Neurología-Neuroinmunología, Centro de Esclerosis Múltiple de Cataluña (Cemcat), Hospital Universitario Vall d'Hebron, Barcelona, Spain

10 Unitat de Neuroimmunología - CSUR Servicio de Neurología Hospital Universitari i Politècnic La Fe de Valencia, Valencia, Spain 
frequently affected cognitive domains are information processing speed (IPS), complex attention, working memory, visuospatial ability, and executive functions [2, 4-8], with predominance of dysexecutive disorders in the progressive forms of MS and an amnestic profile in relapsing-remitting MS [9].

Cognition does not always correlate with the Expanded Disability Status Scale (EDSS) score. CI can be found in early MS $[10,11]$ and also in the asymptomatic forms of MS such as the Radiological Isolated Syndrome (RIS) [2, 3, 12-14]. The presence of CI at the time of diagnosis of MS is considered a poor evolution prognostic marker $[11,15]$. The involvement of verbal memory and IPS, detected in early stages of MS, is predictive of more significant long-term disability. The early detection of CI is of utmost importance in order to ensure a correct social and work adaptation of the patient, and to implement specific cognitive rehabilitation strategies [16].

The Cognition Working Group is part of the EMDAT study group (Multiple Sclerosis Disease Activity Task Force) and is composed by neurologists and a neuropsychologist from different healthcare centers in Spain. The objective of this working group is twofold: to analyze the current difficulties to implement a systematic cognitive assessment (CA) in routine clinical practice and to provide a strategy to detect and monitor cognitive impairment in MS patients based on a systematic review of the literature and the clinical experience of the group.

\section{Cognitive impairment in multiple sclerosis}

\section{Definition}

Fischer and colleagues suggested that, based on the result of a neuropsychological evaluation, CI in MS can be defined by the presence of any of the following aspects [17]:

- A performance below 1.5 or 2 standard deviations (SD) compared to the normative mean in at least $20-30 \%$ of the test parameters.

- A performance below 1.5 or $2 \mathrm{SD}$ in at least two cognitive domains.

It has been considered that these two definitions of CI offer more reliable results [18].

\section{Vulnerability}

The presence and evolution of CI is very heterogeneous among individuals with MS. There is a relationship between magnetic resonance imaging (MRI) data and cognition in MS. However, CI can be only partly explained by the presence of MRI lesions [19], or by other factors such as cognitive reserve (CR) that could explain the discrepancy between MRI and CI. The CR describes the ability to adapt cognitive activity despite brain damage [20] and can be measured with specific test [21] such as the Cognitive Reserve Index Questionnaire (CRIq). Vulnerability of developing CI in MS patients is higher when they present with high lesion load and cerebral atrophy at baseline MRI combined with low scores in CR test and cognitive tests at the beginning of the disease [22].

In order to detect CI earlier and improve prognosis, it has been suggested that the following three aspects should be considered: to perform early and periodic cognitive evaluations, to offer neuropsychological interventionist programs, and to consider the presence of $\mathrm{CI}$ as another possible poor prognostic factor when selecting disease-modifying treatments $[16,23]$.

\section{Importance and utility of cognitive function measurement in multiple sclerosis}

CI has an impact on different aspects of MS patients' daily life functioning [22]. The periodical evaluation of the cognitive function in MS would provide [23]:

- Knowledge of the baseline condition of the patient.

- Information about any cognitive change throughout the disease, either related with disease progression or cognitive relapses [24].

- Prognostic information in order to select a specific disease modifying treatment, as CI is considered a poor prognostic factor with a possible negative impact on treatment adherence.

- Information for the patient and family or caregivers about the presence of the CI that might help them to resolve doubts, and facilitate social work and socio-family adaptation.

- A working scenario to plan an early therapeutic intervention (cognitive rehabilitation and enhancement of the cognitive reserve).

\section{Therapeutic approach to cognitive impairment}

Previous studies have shown that neuropsychological interventions produce positive effects on cognitive performance and other associated abilities, but results from clinical trials are still inconclusive due to methodological limitations [25]. Neuropsychological intervention aims to implement strategies to compensate for or recover cognitive deficits, to promote awareness of CI by the patients and their environment, and to work on the impact that $\mathrm{CI}$ has on their daily life activities.

A Cochrane review of 20 studies about the effects of cognitive rehabilitation concluded that there was a low level of evidence for its recommendation; however, the comparison of the different rehabilitation strategies was difficult because of the heterogeneity of interventions and outcome measures [25]. It was also reported that some studies showed that cognitive 
training improved immediate and working memory abilities and the use of specific rehabilitation training programs did have a positive impact on attention and immediate and verbal memory [25]. In a later review, the results of cognitive intervention on executive function and attention (frequently impaired in MS patients) were more consistent [26].

Functional neuroimaging facilitates the establishment of a correlation between image and cognitive abilities in MS [27] and has been used to study the efficacy of cognitive rehabilitation programs [28]. New research explores the concept of adaptive versus maladaptive neuroplasticity in relation with cognitive rehabilitation programs. Chiaravalloti and colleagues observed that there is an activity increase locally in tissue immediately surrounding a demyelinating lesion, which appears early in MS patients even without CI. This was often associated with intact cognitive functioning and was interpreted as adaptive neuroplasticity. Extra-region activation is considered adaptive inefficiency and is associated with worse cognitive performance [29].

\section{Current status of cognitive assessment: usual difficulties}

We considered that the main limiting factors for $\mathrm{CA}$ in patients with MS are the following:
- Time limitation during the neurologist consultation.

- Lack of material and human resources, especially trained personnel and special equipment $[6,12,30]$.

- Limited availability of neuropsychologists with specific training in MS working in MS centers.

- Lack of validated tests and normative data that account for language differences and different educational levels to obtain reliable results $[2,6]$.

- Limited evidence about therapeutic interventions, since research into the effect of pharmacological treatments on the cognitive status of patients with MS has shown inconsistent results [31].

\section{Tools to measure cognitive impairment in multiple sclerosis}

Neuropsychological tests and questionnaires are tools that allow professionals to analyze and quantify cognitive abilities individually and to compare individuals' performances. Adequate training for administration and interpretation of the neuropsychological testing is mandatory. In MS, some tools have demonstrated a higher sensitivity in the detection of CI and they are the ones used more often. As CI in MS shows a distinctive profile compared to other neurodegenerative diseases, the neuropsychological tools for CA must be specific (Table 1) [32-42].
Table 1 Recommended tests for the evaluation of specific cognitive functions (based on Arnett and Forn, 2007) [32]

\begin{tabular}{ll}
\hline Cognitive domain & Test \\
\hline Orientation & Orientation subtest WMS-III [33] \\
Processing speed & PASAT [34, 35] \\
Sustained attention & CPT [36]SDMT [34] \\
Memory & \\
Immediate verbal memory & Direct Digit Subtest of WMS-III [33] or WAIS- III [37] \\
Verbal working memory & Inverse Digit Subtest of WMS-III [33] or WAIS- III [37] \\
& Letter Number Sequencing subtest of WAIS-III [37] \\
Auditory verbal learning and long-term & Spain/Complutense Verbal learning Test (TAVEC) [38] \\
memory/recall & \\
Executive functioning & \\
Visuospatial learning and visuospatial & 10/36 SPART [34, 35] \\
$\quad$ long-term memory & F, A, S [39] \\
Phonetic verbal fluency & Animals, fruits, vegetables [30] \\
Semantic verbal fluency & Tower of London [40] \\
Planification & WCST [41] \\
Abstract reasoning & Similarities subtest of WAIS-III [37] \\
Abstract verbal reasoning & JLOB [42] \\
Visuospatial functions & \\
\hline
\end{tabular}

WMS-III: Wechsler Memory Scale III; PASAT: Paced Auditory Serial Addition Test; CPT: Continuous Performance Test; SDMT: Symbol Digit Modalities Test; WAIS-III: Wechsler Adult Intelligence Scale III; 10/ 36 SPART, 10/36 Spatial Recall Test; WCST: Wisconsin Card Sorting Test; JLO: Judgement of Line Orientation 
CA relies on both quantitative and qualitative information [43] and the neuropsychological assessment can be performed using either a screening test or a cognitive-specific neuropsychological tool.

\section{Screening tests for cognitive assessment}

Cognitive screening is performed when cognitive status is previously unknown [44]. These tests are brief, simple and with high sensitivity and specificity [45]. The most utilized in MS are the following (see Table 2) [46-49]:

\section{- MSNQ (Multiple Sclerosis Neuropsychological Questionnaire)}

The MSNQ is a questionnaire with 15 items that evaluate the neuropsychological competences in daily life activities, by assessing different cognitive domains (attention, memory, processing speed, etc. [30]). There are two versions of the test: one version for the patient (MSNQ-self report or MSNQ-S) and another one for the caregiver (MSNQ-informant report or MSNQ-I). The usefulness of MSNQ-S is limited since it is highly depended on depression and mood disorders [30]. A cut-off score of $\leq 24$ in MSNQ-S will correctly classify $68 \%$ of the patients, with a sensitivity of 0.83 and a specificity of 0.6 ; a score $>22$ at MSNQ-I will correctly classify $85 \%$ of the patients with a sensitivity of 0.87 and a specificity of 0.84 [49, 50].

The MSNQ-I is useful to identify risk groups. It is not influenced by the patient's depression, and correlates well with working memory, learning, and executive and visuospatial functions [50]. It also correlates with certain MRI parameters (lesion volume and brain atrophy), secondary progressive course, and work disability. It has been shown that an increment of one point in the MSNQ-I increases the risk of CI by $6.5 \%$ [30]. The main advantage of this test is that it is easy, short, and easily reproducible $[12,30,51]$. The main drawbacks are the uncertainty of the informant's depression effect on the score, the poor CI detection capability when patients show low repercussion effects on their daily lives, and the lack of the MSNQ-I score for unaccompanied patients $[12,30]$.

\section{- PASAT 3" (3-s Paced Auditory Serial Addition Task)}

The PASAT 3" is used as a multi-domain measure, informing about complex attention processes, working memory, and executive functions. Single digits are presented every $3 \mathrm{~s}$ and the patient must add each new digit to the one immediately prior to it. It was included in the Multiple Sclerosis Functional Composite (MSFC) as a measure of disease monitoring and to measure cognition in clinical trials [52].

Is a short test $(10-15 \mathrm{~min})$ with good sensitivity to detect $\mathrm{CI}$ in MS patients, offering cognitive information independently of the speed of processing [53]. It can distinguish between healthy controls and MS patients with a cut-off score of 43 (sensitivity of 0.82 and a specificity of 0.69 ) and CI in MS patients with a cut-off score of 40.1 (sensitivity 0.74 and specificity 0.65 ) [54]. Nevertheless, still there is not a general cut-off point to define a clinically significant progression [55], it can be stressful for the patient, usually requires specific material and its outcome depends on different factors, such as education or age. Those are the reasons why it has been replaced in many clinical trials by the SDMT (Symbol Digit Modality Test) [51].

\section{- SDMT (Symbol Digit Modality Test)}

In this test, the patients are shown a visual key that matches numbers and symbols on the top of a sheet.

Table 2 Rapid screening tests to detect cognitive impairment in patients withmultiple sclerosis

\begin{tabular}{|c|c|c|c|}
\hline \multirow[b]{2}{*}{ Domain } & \multicolumn{3}{|l|}{ Test } \\
\hline & PASAT $[46,47]$ & SDMT [48] & $\mathrm{MSNQ}^{\mathrm{a}}[49]$ \\
\hline Auditory processing speed and working memory & + & & \\
\hline Visual processing speed and working memory & & + & \\
\hline Information processing speed & & & + \\
\hline Verbal learning & & & + \\
\hline Mean score (standard deviation) & $46.7(9.1)^{\mathrm{b}} 50.4(9.7)^{\mathrm{c}}$ & Depends on age and years of education & Not applicable \\
\hline Duration & $10-15$ minutes & 90 seconds(5 minutes including instructions) & Self-administered \\
\hline
\end{tabular}

MSNQ: Multiple Sclerosis Neuropsychological Questionnaire; PASAT: Paced Auditory Serial Addition Test; SDMT: Symbol Digit Modalities Test

${ }^{a}$ MSNQ-Informant report correlates with Boston Naming Test $(-0.45, \mathrm{p}<0.001)$, CVLT-II Total Recall Trials 1-5 (-0.53, p<0.01), CVLT-II Delayed Recall (-0.43, $\mathrm{p}<0.001)$, BVMT-R Delayed Recall (-0.43, $\mathrm{p}<0.001)$, Trail Making Test $(0.55, \mathrm{p}<0.01)$, Paced Auditory Serial Addition $(-0.47, \mathrm{p}<0.001)$, WCST Perseveration Responses $(0.37, \mathrm{p}<0.01)$

${ }^{\mathrm{b}}$ Scores up to 12 years of education and ${ }^{\mathrm{c}}$ scores for 12 or more years of education, according to Rao et al (1991) [7] 
Then, they must specify the correct number for each of the symbols presented as fast and as accurately as they can during $90 \mathrm{~s} \mathrm{[51].} \mathrm{This} \mathrm{test} \mathrm{assesses} \mathrm{processing} \mathrm{speed}$ and visual working memory. The SDMT is fast, easy, and has a low cost $[2,3]$. It is sensitive for the detection and change of CI in MS [6]. The score of the test correlates with CI, MRI (lesion load, ventricular volume, cortical, and deep grey matter atrophy), and with the patient's functional status $[2,6]$. The SDMT has a higher capacity in distinguishing patients with MS from controls. Of the cognitive tests included in the BRB-N (Rao Brief Repeatable Neuropsychological Battery) and the MACFIMS (Minimal Assessment of Cognitive Function in Multiple Sclerosis), the SDMT is the best test for the detection of CI in MS $[6,12,35,56]$. An optimal cut-off score of 55 hits has been suggested, correctly categorizing $72 \%$ of patients, with a sensitivity of 0.82 , specificity of 0.60 , and a positive predictive value (PPV) of 0.71 [49, 50]. A decrement of 4 or more points or $10 \%$ of the SDMT or 0.5 SD could be considered a clinically significant measure of cognitive worsening [57]

This is a fast test $(5 \mathrm{~min})$, easily reproducible, and does not require specific neuropsychological training for its administration $[2,51]$. Compared to the other tests, it is more sensitive [57], requires less time, does not require any electronic equipment, and has a prognostic value correlating with the disability degree at 5 and 7 years $[2,13,51]$. As a drawback, it is just a screening test that does not offer any information about other cognitive domains.

\section{Neuropsychological evaluation batteries}

A more extensive neuropsychological evaluation allows for a more detailed study of several cognitive domains. The use of neuropsychological batteries is recommended after either a positive screening test, subjective cognitive complaints reported by the patient or his caregiver, discordance between clinical perception/screening tests or in specific socio-labor situations $[16,18]$. The contents of the three most used neuropsychological batteries in MS are detailed in Table $3[6,58,59]$.

\section{Brief International Cognitive Assessment For Multiple Sclerosis (BICAMS)}

It is a brief battery to be used as screening of cognitive status that can be administered by health personnel without specific training in about $15 \mathrm{~min}$. It evaluates processing information speed with the SDMT, verbal memory with the CVLT-II (California Verbal Learning Test), and visual memory based on the result of the BVMT-R (Brief Visuospatial Memory Test revised), excluding scales of executive and visuospatial functions. If the assessment time is limited, the authors recommend to shorten the BICAMS and administer only the SDMT component.

CVLT-II evaluates learning and memory of verbal material. From all the indexes that this test offers, the BICAMS authors suggest the use of only the first five learning trials because this reduces time and possible effects of fatigue. This way of execution has been validated with MRI and brain atrophy measurements [58, 60, 61].

The BVMT-R assesses visual learning and memory. The protocol suggested in the BICAMS includes only the first three recall trials given their significant relationship with MRI parameters, brain atrophy [58,60,61], and functioning of diencephalic nuclei $[60,62]$. It has shown a greater discriminative validity and sensitivity for visuospatial memory, with the advantage that it does not require specialized material and has no ceiling effect, although the functionality of the upper limbs may influence its outcomes $[12,56]$.

\section{Brief Repeatable Battery-Neuropsychology (BRB-N)}

This battery is composed by the SDMT, the PASAT 3", the Selective Reminder Test (SRT), the 10/36 Spatial Recall Task (SPART), and the Word List Generation (WLG). The SRT is a verbal learning and memory test, the SPART assesses visual learning and memory, and the WLG assesses verbal fluency. In theory, the BRB-N is administered in 30-35 min although, depending on the patient's capacity, it can take longer. Its administration and interpretation require a professional with training in neuropsychology. It has high sensitivity (67-71\%) and specificity (85-94\%). The BRB-N has an alternative version to avoid learning bias [34] and is available in Spanish [35]. As a disadvantage, some cognitive functions are poorly evaluated, or excluded, increasing the risk of false negatives.

\section{Minimal Assessment of Cognitive Function in Multiple Sclerosis (MACFIMS)}

It has been considered an improved version of the BRB-N [56] as it evaluates all cognitive functions affected in MS. In the MACFIMS visuospatial function tasks such as the Benton Line Orientation Judgment (JOLB), the PASAT paced every $2 \mathrm{~s}$ and the Denis-Kapplan Battery (D-KEFS) Chart Classification trial for executive functioning were added.

Modifications to verbal memory measures are introduced, including the California Learning Verbal Test III, which replaced the SRT; the measurement of visual memory is changed to the Brief Visuospatial Memory Test-Revised (BVMT-R); and the measure of language fluency to the COWAT. It is administered in about $90 \mathrm{~min}$. Adequate training in clinical neuropsychology is required [58]. 


\section{Recommendations and suggestions from the EMDAT group}

Different factors should be considered when performing the $\mathrm{CA}$, such as CR, emotional status (anxiety and/or depression), fatigue, and concomitant pharmacotherapy [16].

\section{When and where to perform cognitive assessment}

CA should be performed in a quiet place without distractions. It is not recommendable to perform the assessment during the following month after a relapse or corticosteroid treatment, as they have a detrimental effect on memory [6]. It is convenient to conduct the assessments at the same time of the day to avoid possible biases of fatigue during the follow-up. Initially, it should be performed annually or biannually, and then, according to clinical criteria. It is necessary to take into account that certain medications may influence cognitive performance and also the patient mood $[16,18]$. It is recommended that the baseline evaluation is performed as soon as possible after the diagnosis of MS, with subsequent periodic follow-ups.

\section{Neuropsychological evaluation according to available recourses in different healthcare centers (Fig. 1)}

Availability of time and human resources varies between different hospitals, which may interfere with a correct CA. It is suggested that all patients should have a baseline and annual screening test completed, and a baseline and periodic neuropsychological evaluation should be performed on all patients at a suggestion level. Recommendation for follow-up evaluations should be made for any of the following scenarios: (i) a positive screening test; (ii) a negative screening result but with a subjective cognitive complaint reported either by the patient or family members;

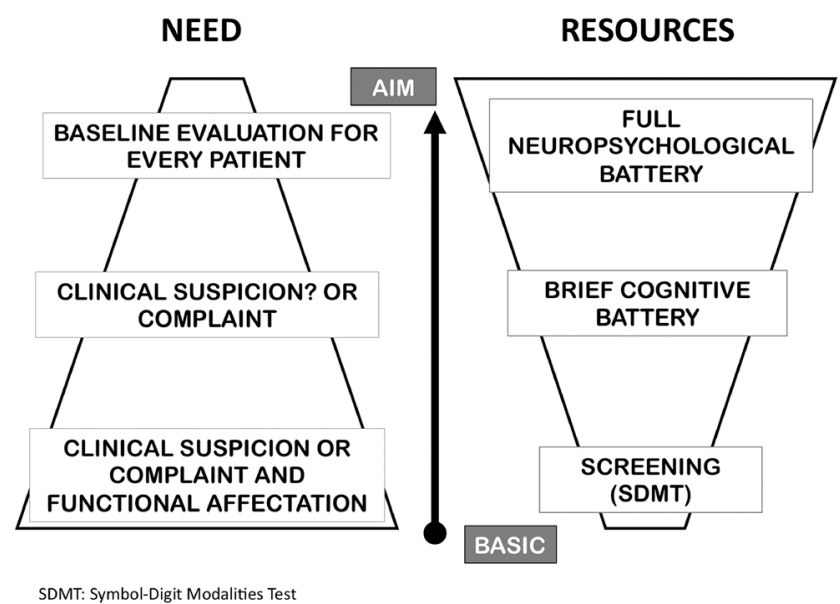

Fig. 1 Description of current reality considering factors of need and available resources

(iii) worsening of cognitive impairment that will require a complete evaluation; (iv) any social or working negative impact.

\section{Centers with an available neuropsychologist in their staff}

We recommend the BRB-N battery, and the addition of specific questionnaires to measure mood (BDI-II or HADS), fatigue (MSFIS), and quality of life (MSQoL-54) (See Table 4).

\section{Centers with limited human resources}

If there is limited access or no availability of a neuropsychologist or neurologist with specific training, or there is limited time for each patient, the SDMT test is recommended as cognitive screening. If health caregivers with specific training are available, it is suggested to also administer the MSQN. A

Table 3 Neuropsychological batteries for the evaluation of patients withmultiple sclerosis

\begin{tabular}{|c|c|c|c|}
\hline \multirow[b]{2}{*}{ Domain } & \multicolumn{3}{|l|}{ Batteries } \\
\hline & MACFIMS [58] & BRNB [59] & BICAMS [6] \\
\hline Auditory processing speed and working memory & PASAT & PASAT & \\
\hline Visual processing speed and working memory & SDMT & SDMT & SDMT \\
\hline Verbal/auditory memory & CVLT2 & SRT & CVLT2 \\
\hline Visual/spatial memory & BVMTR & 10/36 SPART & BVMTR \\
\hline Language & COWAT & COWAT & \\
\hline Spatial processing & JLO & & \\
\hline Executive functioning & DKEFS Sorting & & \\
\hline Duration & 90 minutes & 50 minutes & 20 minutes \\
\hline
\end{tabular}

CVLT2: California Verbal Learning Test second edition; SRT: Selective Reminding Test; BVMTR: Brief Visuospatial Memory Test Revised; 10/36 SPART: 10/36 Spatial Recall Test; COWAT: Controlled Oral Word Association Test; JLO: Judgement of Line Orientation; DKEFS: Delis-Kaplan Executive Function System 


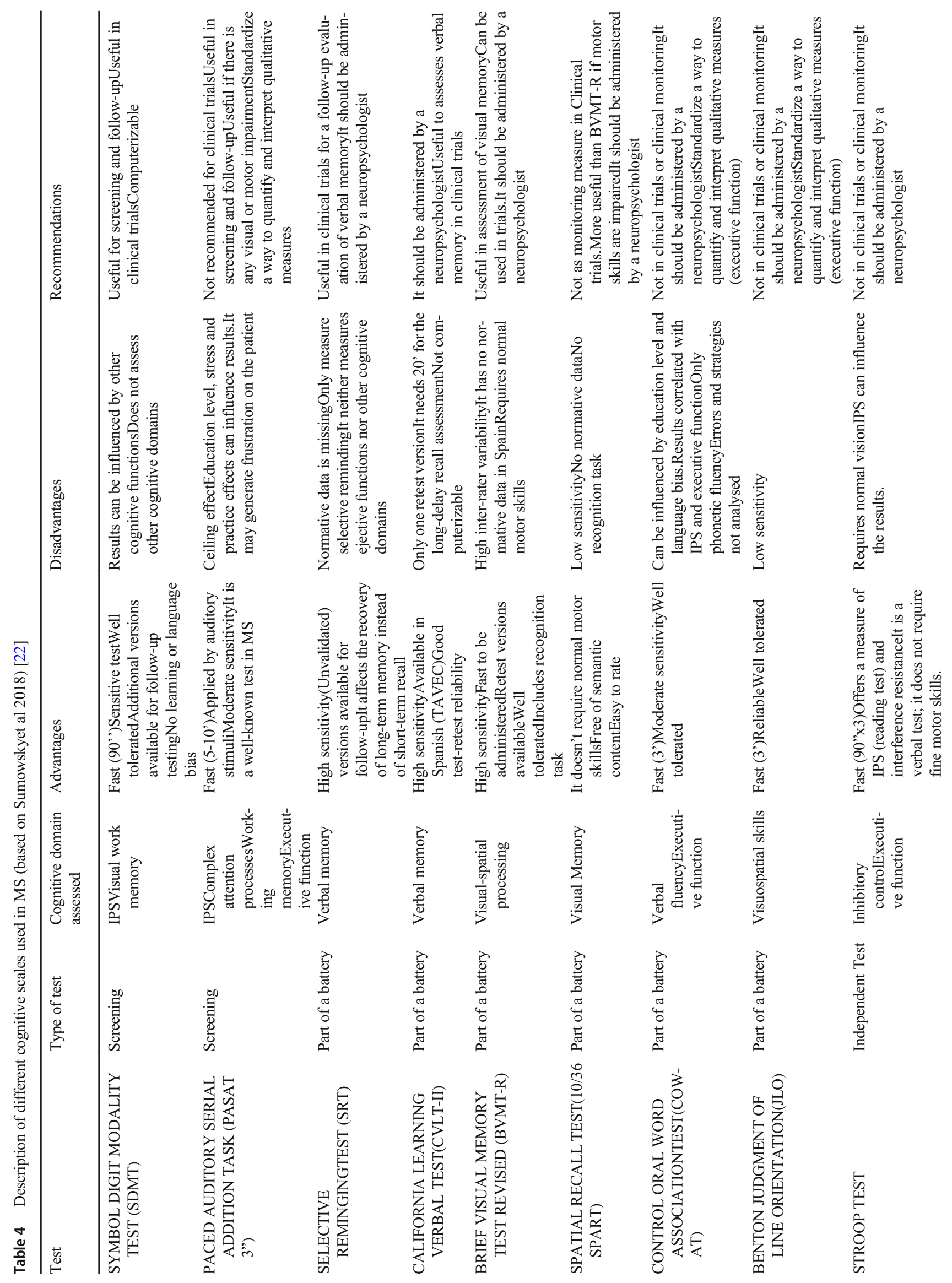


complete neuropsychological evaluation is recommended with abnormal SDMT scores [40-43] or when this score diminishes a $10 \%$ of the normal value or a score of 4 points below previous SDMT values. The cognitive screening test should neither replace a neuropsychological assessment nor be used for diagnosis since this might produce an increase of false negatives $[3,12]$.

\section{Evolution and follow-up}

In case of normal results in cognitive screening tests or in cases with mild CI, no repercussion on daily life activities, and not classifiable as dementia [63], an annual evaluation is recommended for the first 2 years of follow-up, and thereafter depending on clinical evolution. In case of moderate or severe cognitive impairment, annual or biannual evaluations are recommended. After that they should be spaced out according to clinical criteria, until the patient meets the criteria for dementia, in which case stopping them is suggested. Intermediate evaluations are recommended if cognitive changes are noted.

\section{Next challenges}

Multiple tools for a complete CA require specialized training in cognition, and they need to be carried out by a multidisciplinary team. The incorporation of neuropsychologists in Neurology Services and specifically in MS Units would improve patient care. It is necessary to standardize cognitive tests used in MS to homogenously quantify cognitive impairment. Causes of inter-patient cognitive variability are unknown [22]; therefore, analysis of cases with brain damage documented by MRI but normal neuropsychological results become important. It has been observed that MS patients with a high educational level in the initial phases of the disease have similar neuropsychological results compared to healthy controls the only exception being the processing speed [13]. This fact could suggest that compensating mechanisms are less efficient in protecting against deterioration of IPS; therefore, it would be essential to investigate how to empower them. There are critical radiological biomarkers such as brain atrophy related to CI [3], but they are not generally available in clinical practice. It has been described that the anti-inflammatory effect of some immunomodulation drugs could be beneficial in cognition [64]. However, there is no evidence that patients with CI benefit from a specific disease-modifying treatment, or from a change in the treatment. Finally, given that MS is a very complex disease, it would be ideal to have objective risk markers to identify patients with MS at risk of developing CI before presenting it. 


\section{Conclusions}

Given that $\mathrm{CI}$ in MS is frequent and represents a prognostic factor for disability in the long term, a complete CA must be performed on all patients at the time of diagnosis. CA allows informing and advising patients to facilitate their daily life, as well as to promote and design rehabilitation strategies for cognitive stimulation. It is necessary to include specialized professionals in the multidisciplinary EM teams to optimize the evolution and follow-up of patients.

Acknowledgements The authors would like to thank Blanca Gonzalez y Marta Ferrando.

Author contribution All authors have approved the manuscript and contributed sufficiently to the preparation of the document.

\section{Declarations}

Ethical approval This is a paper without patient implication. Informed consent is not necessary.

Conflict of interest The authors declare no competing interests.

Open Access This article is licensed under a Creative Commons Attribution 4.0 International License, which permits use, sharing, adaptation, distribution and reproduction in any medium or format, as long as you give appropriate credit to the original author(s) and the source, provide a link to the Creative Commons licence, and indicate if changes were made. The images or other third party material in this article are included in the article's Creative Commons licence, unless indicated otherwise in a credit line to the material. If material is not included in the article's Creative Commons licence and your intended use is not permitted by statutory regulation or exceeds the permitted use, you will need to obtain permission directly from the copyright holder. To view a copy of this licence, visit http://creativecommons.org/licenses/by/4.0/.

\section{References}

1. Rao SM, Leo GJ, Ellington L, Nauertz T, Bernardin L, Unverzagt F (1991) Cognitive dysfunction in multiple sclerosis. II. Impact on employment and social functioning. Neurology 41(5):692-696

2. Van Schependom J, D'hooghe MB, Cleynhens K et al (2014) The Symbol Digit Modalities Test as sentinel test for cognitive impairment in multiple sclerosis. Eur J Neurol 21(9):1219-1225

3. Deloire MSA, Bonnet MC, Salort E, Arimone Y, Boudineau M, Petry KG, Brochet B (2006) How to detect cognitive dysfunction at early stages of multiple sclerosis? Mult Scler 12(4):445-452

4. Labiano-Fontcuberta A, Mitchell AJ, Moreno-García S, BenitoLeón J (2014) Cognitive impairment in patients with multiple sclerosis predicts worse caregiver's health-related quality of life. Mult Scler J 20(13):1769-1779

5. Chiaravalloti ND, DeLuca J (2008) Cognitive impairment in multiple sclerosis. Lancet Neurol 7(12):1139-1151

6. Langdon DW, Amato MP, Boringa J, Brochet B, Foley F, Fredrikson S, Hämäläinen P, Hartung HP, Krupp L, Penner IK, Reder AT, Benedict RHB (2012) Recommendations for a Brief
International Cognitive Assessment for Multiple Sclerosis (BICAMS). Mult Scler J 18(6):891-898

7. Rao SM, Leo GJ, Bernardin L, Unverzagt F (1991) Cognitive dysfunction in multiple sclerosis. I. Frequency, patterns, and prediction. Neurology 41(5):685-691

8. Rocca MA, Amato MP, De Stefano N et al. MAGNIMS Study Group (2015). Clinical and imaging assessment of cognitive dysfunction in multiple sclerosis. Lancet Neurol 14(3):302-317

9. Zakzanis KK (2000) Distinct neurocognitive profiles in multiple sclerosis subtypes. Arch Clin Neuropsychol 15(2):115-136

10. Feinstein A, Kartsounis LD, Miller DH, Youl BD, Ron MA (1992) Clinically isolated lesions of the type seen in multiple sclerosis: a cognitive, psychiatric, and MRI follow up study. J Neurol Neurosurg Psychiatry 55(10):869-876

11. Glanz BI, Holland CM, Gauthier SA et al (2006) Cognitive dysfunction in patients with clinically isolated syndromes or newly diagnosed multiple sclerosis. Mult Scler J 13(8):1004-1010

12. Benedict RHB, Zivadinov R (2006) Predicting neuropsychological abnormalities in multiple sclerosis. J Neurol Sci 245(1-2):67-72

13. Deloire M, Ruet A, Hamel D, Bonnet M, Brochet B (2010) Early cognitive impairment in multiple sclerosis predicts disability outcome several years later. Mult Scler 16(5):581-587

14. Labiano-Fontcuberta A, Martínez-Ginés ML, Aladro Y, Ayuso L, Mitchell AJ, Puertas-Martín V, Cerezo M, Higueras Y, BenitoLeón J (2016) A comparison study of cognitive deficits in radiologically and clinically isolated syndromes. Mult Scler J 22(2):250-253

15. Zipoli V, Goretti B, Hakiki B, Siracusa G, Sorbi S, Portaccio E, Amato MP (2010) Cognitive impairment predicts conversion to multiple sclerosis in clinically isolated syndromes. Mult Scler J 16(1):62-67

16. Kalb R, Beier M, Benedict RHB, Charvet L, Costello K, Feinstein A, Gingold J, Goverover Y, Halper J, Harris C, Kostich L, Krupp L, Lathi E, LaRocca N, Thrower B, DeLuca J (2018) Recommendations for cognitive screening and management in multiple sclerosis care. Mult Scler J 24(13):1665-1680

17. Fischer M, Kunkel A, Bublak P, Faiss JH, Hoffmann F, Sailer M, Schwab M, Zettl UK, Köhler W (2014) How reliable is the classification of cognitive impairment across different criteria in early and late stages of multiple sclerosis? J neurol sci 343(1):91-99

18. Amato MP, Morra VB, Falautano M, Ghezzi A, Goretti B, Patti F, Riccardi A, Mattioli F (2018) Cognitive assessment in multiple sclerosis-an Italian consensus. Neurol Sci 39(8):1317-1324

19. Benedict RH, Zivadinov R (2011) Risk factors for and management of cognitive dysfunction in multiple sclerosis. Nat Rev Neurol 7(6):332-342

20. Stern Y (2002) What is cognitive reserve? Theory and research application of the reserve concept. J Int Neuropsychol Soc 8(3): 448-460

21. Nucci M, Mapelli D, Mondini S (2012) Cognitive Reserve Index questionnaire (CRIq): a new instrument for measuring cognitive reserve. Aging Clin Exp Res 24(3):218-226

22. Sumowski JF, Leavitt VM, Rocca MA, Inglese M, Riccitelli G, Buyukturkoglu K, Meani A, Filippi M (2018) Mesial temporal lobe and subcortical grey matter volumes differentially predict memory across stages of multiple sclerosis. Mult Scler J 24(5):675-678

23. Cheng EM, Crandall CJ, Bever CT Jr, Giesser B, Haselkorn JK, Hays RD, Shekelle P, Vickrey BG (2010) Quality indicators for multiple sclerosis. Mult Scler J 16(8):970-980

24. Pardini M, Uccelli A, Grafman J, Yaldizli O, Mancardi G, Roccatagliata L (2014) Isolated cognitive relapses in multiple sclerosis. J Neurol Neurosurg Psychiatry 85:1035-1037 
25. Rosti-Otajärvi EM, Hämäläinen PI (2014) Neuropsychological rehabilitation for multiple sclerosis. Cochrane Database Syst Rev 2: CD009131

26. Mitolo M, Venneri A, Wilkinson ID, Sharrack B (2015) Cognitive rehabilitation in multiple sclerosis: a systematic review. J Neurol Sci 354(1):1-9

27. DeLuca GC, Yates RL, Beale H, Morrow SA (2015) Cognitive impairment in multiple sclerosis: clinical, radiologic and pathologic insights. Brain Pathol 25(1):79-98

28. Tomassini V, Matthews PM, Thompson AJ (2012) Neuroplasticity and functional recovery in multiple sclerosis. Nat Rev Neurol 8(11):635-646

29. Chiaravalloti ND, Genova HM, DeLuca J (2015) Cognitive rehabilitation in multiple sclerosis: the role of plasticity. Front Neurol 6:67

30. O'Brien A, Gaudino-Goering E, Shawaryn M et al (2007) Relationship of the Multiple Sclerosis Neuropsychological Questionnaire (MSNQ) to functional, emotional, and neuropsychological outcomes. Arch Clin Neuropsychol 22:933-948

31. Miller E, Morel A, Redlicka J, Miller I, Saluk J (2018) Pharmacological and non-pharmacological therapies of cognitive impairment in multiple sclerosis. Curr Neuropharmacol 16(4):475483

32. Arnett P, Forn C (2007) Neuropsychological evaluation in multiple sclerosis. Rev Neurol 44(3):166-172

33. Wechsler D (2004) Wechsler Memory Scale III. TEA Ediciones, Madrid

34. Boringa JB, Lazeron RHC, Reuling JEW et al (2001) The brief repeatable battery of neuropsychological tests: normative values allow application in multiple sclerosis clinical practice. Mult Scler 7:263-267

35. Sepulcre J, Vanotti S, Hernández R, Sandoval G, Cáceres F, Garcea O, Villoslada P (2006) Cognitive impairment in patients with multiple sclerosis using the Brief Repeatable Battery-Neuropsychology test. Mult Scler J 12(2):187-195

36. Conners CK (2000) Conners' Continuous Performance Test user's manual. Multi-Health Systems, Toronto, Canada

37. Wechsler D, Kaufman AS, Lichtenberger EO (2001) Wechsler Adult Intelligence Scale III. TEA Ediciones, Madrid

38. Benedet MJ, Alejandre MA (1998) Test de Aprendizaje Verbal España-Complutense (TAVEC). TEA Ediciones, Madrid

39. Buriel Y, Gramunt N, Böhm P et al (2004) Verbal fluency: preliminary normative data in a Spanish sample of young adults (20-49 years of age). Neurologia 19(4):153-159

40. Krikorian R, Bartok J, Gay N (1994) Tower of London procedure: a standard method and developmental data. J Clin Exp Neuropsychol 16(6):840-850

41. Heaton RK (2001) Test de clasificación de tarjetas de Wisconsin. TEA Ediciones, Madrid

42. Peña-Casanova J, Gramunt-Fombuena N, Gich-Fullà J (2004) Test de orientación de líneas de Benton. In: Peña-Casanova J, GramuntFombuena N, Gich-Fullà $J$ (eds) Test neuropsicológicos. Fundamentos para una neuropsicología clínica basada en evidencias. Masson, Barcelona, pp 227-230

43. Lezak MD, Howieson DB, Bigler ED, Tranel D (2012) Neuropsychological Assessment. New York Oxford University Press, OUP USA

44. Scherer P (2007) Cognitive screening in multiple sclerosis. J Neurol 254(2):II $26-$ II 29

45. Deeks JJ (2001) Systematic reviews in health care: systematic reviews of evaluations of diagnostic and screening tests. BMJ 323: 157-162

46. Sampson $\mathrm{H}$ (1958) Serial addition as a function of stimulus duration and pacing. Can J Psychol 12:179-183
47. Sampson H, MacNeilage PF (1960) Temporal integration and the serial addition paradigm. Aust J Psychol 12:70-88

48. Parmenter BA, Weinstock-Guttman B, Garg N, Munschauer F, Benedict RHB (2007) Screening for cognitive impairment in multiple sclerosis using the Dymbol digit Modalities Test. Mult Scler 13(1):52-57

49. Benedict RHB, Munschauer F, Linn R, Miller C, Murphy E, Foley F, Jacobs L (2003) Screening for multiple sclerosis cognitive impairment using a self-administered 15 -item questionnaire. Mult Scler 9(1):95-101

50. Benedict RH, Cox D, Thompson LL et al (2004) Reliable screening for neuropsychological impairment in multiple sclerosis. Multiple Sclerosis Journal 10(6):675-678

51. Benedict RHB, Duquin JA, Jurgensen S, Rudick RA, Feitcher J, Munschauer FE, Panzara MA, Weinstock-Guttman B (2008) Repeated assessment of neuropsychological deficits in multiple sclerosis using the Symbol Digit Modalities Test and the MS Neuropsychological Screening Questionnaire. Mult Scler 14(7): 940-946

52. Rudick RA, Cutter G, Reingold S (2002) The multiple sclerosis functional composite: a new clinical outcome measure for multiple sclerosis trials. Mult Scler J 8(5):359-365

53. Sonder JM, Burggraaff J, Knol DL, Polman CH, Uitdehaag BMJ (2014) Comparing long-term results of PASAT and SDMT scores in relation to neuropsychological testing in multiple sclerosis. Mult Scler J 20(4):481-488

54. Rosti E, Hämäläinen P, Koivisto K, Hokkanen L (2007) PASAT in detecting cognitive impairment in relapsing-remitting MS. Applied neuropsychology 14(2):101-112

55. Karabudak R, Dahdaleh MP, Aljumah MA, Alroughani R, Alsharoqi IA, AlTahan AM, Bohlega SA, Daif A, Deleu D, Amous A, Inshasi JS, Rieckmann P, Sahraian MA, Yamout BI (2015) Functional clinical outcomes in multiple sclerosis: current status and future prospects. Mult Scler Relat Disord 4(3):192-201

56. Strober L, Englert E, Munschauer F et al (2009) Sensitivity of conventional memory tests in multiple sclerosis: comparing the Rao Brief Repeatable Neuropsychological Battery and the Minimal Assessment of Cognitive Function in MS. Mult Scler 15:1077-1084

57. Benedict RH, DeLuca J, Phillips G et al (2017) Validity of the Symbol Digit Modalities Test as a cognition performance outcome measure for multiple sclerosis. Multiple Sclerosis Journal 23(5): 721-733

58. Benedict RH, Fischer JS, Archibald CJ et al (2002) Minimal neuropsychological assessment of MS patients: a consensus approach. Clin Neuropsychol 16(3):381-397

59. Rao SM (1990) The Cognitive Function Study Group of the National Multiple Sclerosis Society. In: A manual for the brief repeatable battery of neuropsychological tests in multiple sclerosis. Medical College of Wisconsin, Milwaukee, WI

60. Houtchens MK, Benedict RHB, Killiany R, Sharma J, Jaisani Z, Singh B, Weinstock-Guttman B, Guttmann CRG, Bakshi R (2007) Thalamic atrophy and cognition in multiple sclerosis. Neurology 69(12):1213-1223

61. Fink F, Eling P, Rischkau E, Beyer N, Tomandl B, Klein J, Hildebrandt H (2010) The association between California Verbal Learning Test performance and fibre impairment in multiple sclerosis: evidence from diffusion tensor imaging. Mult Scler J 16(3): 332-341

62. Ramasamy DP, Benedict RH, Cox JL et al (2009) Extent of cerebellum, subcortical and cortical atrophy in patients with MS: a casecontrol study. J Neurol Sci 282(1-2):47-54 
63. Gauthier S, Reisberg B, Zaudig M, Petersen RC, Ritchie K, Broich K, Belleville S, Brodaty H, Bennett D, Chertkow H, Cummings JL, de Leon M, Feldman H, Ganguli M, Hampel H, Scheltens P, Tierney MC, Whitehouse P, Winblad B (2006) Mild cognitive impairment. Lancet 367(9518):1262-1270

64. Huijbregts SCJ, Kalkers NF, de Sonneville LMJ, de Groot V, Polman CH (2006) Cognitive impairment and decline in different MS subtypes. J Neurol Sci 245(1-2):187-194
This manuscript is original and has not been previously published or submitted for consideration in other journals.

Publisher's note Springer Nature remains neutral with regard to jurisdictional claims in published maps and institutional affiliations. 